\title{
A FORTRAN IV PROGRAM GENERALIZING THE SCHÖNEMANN-CARROLL MATRIX FITTING ALGORITHM TO MONOTONE AND LINEAR FITTING OF CONFIGURATIONS ${ }^{1}$
}

\author{
JAMES C. LINGOES
}

The University of Michigan

Schönemann and Carroll (1970) proposed a generalization of the orthogonal Procrustes problem for obtaining a least-squares fit of a given matrix $X$ to a target matrix $Y$ under a choice of an orthogonal rotation, a translation, and a central dilation. Such linear displacements leave invariant the relative magnitudes of interpoint distances and the monotone measures of goodness of fit. Lingoes and Schönemann (1973) suggested alternative measures of fit which would permit comparisons among any number of such fitted pairs of matrices. Since a number of recent techniques for data analysis impose only ordinal restrictions for obtaining configurations, it would be desirable to extend the Schönemann-Carroll algorithm to monotone displacements, so that one could more clearly "see" how similar are pairs of matrices. For example, one may have multiple solutions for the same data (based on either different samples or different techniques) or one may have multiple hypothesis matrices, which are to be fitted. It is quite possible in such comparisons to reach a conclusion that a given pair of matrices docs not fit very well, if only linear transformations were used, but to come to the opposite conclusion if one were permitted also to map monotonically $X$ into $Y$.

It will be recalled that the problem posed by Schönemann and Carroll was to determine the

1 This research in nonmetric methods is supported in part by a grant from the National Science Foundation (GS-2850). 


$$
\min _{c, T, \gamma} e=\operatorname{trace}\left(E^{\prime} E\right)
$$

in

$$
Y=c X T+J \gamma^{\prime}+E,
$$

where $J$ is a $n \times 1$ column vector of ones, $X$ and $Y$ are two known $n$ $\times m$ matrices, $T$ is a $m \times m$ transformation matrix for orthogonal rotation, $\gamma^{\prime}$ is the $1 \times m$ translation vector, $c$ is the multiplying scalar for contraction/dilation, and $E$ is the residual $n \times m$ error matrix for $n$ points and $m$ dimensions. The solution for $c, T$, and $\gamma$, which is detailed in Schönemann and Carroll (1970), appears in abbreviated form in Lingoes and Schönemann (1973). The "best fit" matrix is given by

$$
\hat{P}=c X T+J \gamma^{\prime}
$$

To achieve monotonic transformations it will be necessary to re-cast the problem as one of finding

$$
\min _{|D|} Q^{*}=\sum_{i>i}\left(d_{i ;}-d_{i j}^{*}\right)^{2}
$$

for

$$
d_{i i}=\left[\sum_{a=1}^{m}\left(x_{i a}-x_{i a}\right)^{2}\right]^{1 / 2},
$$

the set of Euclidean distances (D) derived from the to-be-fitted matrix $X$. The $\left\{D^{*}\right\}$, on the other hand, represent a set of values monotone with $\{D\}$, i.e., Guttman's (1968) rank-images, which, contrary to the usual practice, are derived from $Y$, the target matrix, by permuting the distances from $Y$ into the order of $\{D\}$. To insure a comparable metric and to increase the rate of convergence, the restriction is imposed that

$$
\sum_{i>i} d_{i i}{ }^{2}=\sum_{i>i} d_{i j}{ }^{2}
$$

for the initial distances from $X$. The important additional constraint is made that $D^{(t)}$ is monotone with $D^{(t=0)}$ for every iteration $t$.

To solve for a set of coordinates which will yield a set of distances suitably transformed so that $Q^{*}$ is minimized requires an iterative procedure where $\left\{D^{*}\right\}$ is fixed and known, while $\{D\}$ varies and is unknown. The Guttman-Lingoes' first-phase algorithm (Guttman, 1968; Lingoes and Roskam, 1973) is ideally suited for this nonlinear problem because of its robustness and nice convergence properties. For the particular $\left\{D^{*}\right\}$, the algorithm yields a global and not merely 
a local minimum. If the distances from the $Y$ configuration are a monotonic function of the distances from the $X$ configuration, then minimizing $Q^{*}$ followed by minimizing $e$ (or equivalently the LingoesSchönemann symmetric measure $S$ ) will yield $\hat{Y}=Y$.

The following notation will be helpful for stating some facts: let $X^{*}$ represent the coordinate set that minimizes $Q^{*}$ when $X$ is to be fitted to $Y$, and, similarly, let $Y^{*}$ be the coordinates that minimize $Q^{*}$ when $Y$ is to be fitted to $X$. Then, although $S(X, Y)=S(Y, X)$, it is not true in general that $S\left(X^{*}, Y\right)=S\left(Y^{*}, X\right)$, unless $X$ and $Y$ are perfect monotonic functions of each other. Nor is it necessarily true that $S\left(X^{*}, Y\right)<S(X, Y)$; i.e., linear transformations may yield a closer fit than if they are preceded by monotonic transformations. The foregoing observations would suggest that for every pair of matrices it will be necessary to compute $S(X, Y), S\left(X^{*}, Y\right)$, and $S\left(Y^{*}, X\right)$ in order to choose the closest matching pair. In comparing multiple solutions an unwanted asymmetry may be introduced by the monotonic displacements unless the target matrix remains fixed.

As an illustration the reader can refer to the data given in Tables 2 and 3 of Schönemann and Carroll (1970, p. 251ff). For the configurations in Table $2, Q^{*}$ vanished and $S\left(X^{*}, Y\right)=S\left(Y^{*}, X\right)=0$, which contrasts with $S(X, Y)=.012$, representing approximately an 11 per cent reduction in error of fit. The comparable values for Table 3, on the other hand, were: $.243, .247$, and .249 , respectively, if one substitutes $X$ for their matrix $A$ and $Y$ for $B$. Thus, while some improvement was brought about by monotonic transformations, none of these improvements represented more than a one per cent reduction in error of fit over that obtained by a linear transformation. It is suspected that as $n$ increases for a given $m$, there will be less and less room for monotonic improvement in fit. Furthermore, as the dimensionality of $X$ and $Y$ became more discrepant (regardless of $n$ ), more will be gained by performing the monotone transformation.

Capacity

$4 \leq n \leq 100 ; m \leq 10$; for 159,600 bytes of storage.

\section{Availability}

For this and 50 other programs and subroutines in the $G-L$ Series (covering 23 nonmetric models) see Lingoes (1973). For free litera- 
ture write to: Prof. James C. Lingoes, Computing Center Annex, 1000A North University Building, The University of Michigan, Ann Arbor, Michigan, 48104.

\section{REFERENCES}

Guttman, L. A general nonmetric technique for finding the smallest coordinate space for a configuration of points. Psychometrika, $1968,33,469-506$.

Lingoes, J. C. The Guttman-Lingoes Nonmetric Program Series. Mathesis Press, Ann Arbor, Michigan, 1973.

Lingoes, J. C. and Roskam, E. A mathematical and empirical analysis of two multidimensional scaling algorithms. Psychometric Monographs, $1973,38$.

Lingoes, J. C. and Schönemann, P. H. Alternative measures of fit for the Schönemann-Carroll matrix fitting algorithm. Michigan Mathematical Psychology Program, 1973, 6, 1-6. Also in: Psychometrika, 1974, 39, in press.

Sehönemann, P. H. and Carroll, R. M. Fitting one matrix to another under choice of a central dilation and a rigid motion. Psychometrika, 1970, 35, 245-255. 